\title{
Long-term Survival after Combined Epidural-General Anesthesia or General Anesthesia Alone: Follow-up of a Randomized Trial
}

Du YT, Li YW, Zhao BJ et al. Anesthesiology 2021; 135:233-45

$\mathrm{D}$ ie Inzidenzrate von Krebserkrankungen und die damit verbundene Mortalität steigen trotz moderner Therapiemöglichkeiten leider weiterhin an. Laut WHO weisen Krebserkrankungen die zweithöchste Todesrate weltweit auf. Dabei ist die Todesursache bei Krebs oft auf die Entstehung von Metastasen zurückzuführen. Ob und wann Metastasen entstehen, hängt wiederum von der Balan-

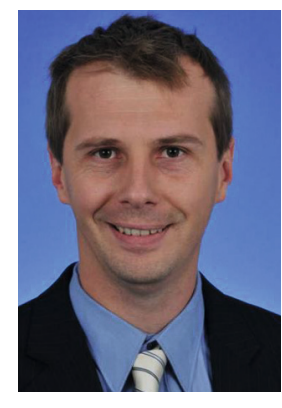

Von

Prim. Doz. Dr. Johann Knotzer, MSc

Leiter der Abteilung für Anästhesiologie und Intensivmedizin am Klinikum WelsGrieskirchen

Inhalationsanästhetika, Opiate und Antiemetika. Dafür brauchte diese Patientengruppe signifikant mehr Vasopressoren und kolloidale Flüssigkeit. Die Kombinationsgruppe hatte einen niedrigeren mittleren arteriellen Blutdruck, eine höhere Herzfrequenzrate und eine höhere Harnproduktion. Die Inzidenz eines postoperativen Delirs war in der Kombinationsgruppe deutlich geringer.

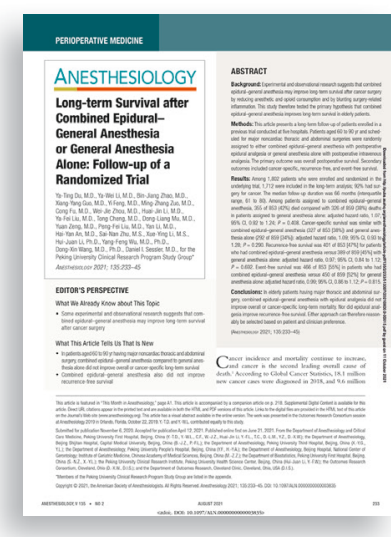

ce zwischen

Aggressivität des primären Tumors und dem körpereigenen Abwehrsystem - hier in erster Linie von den NK(Natural Killer)Zellen - ab. Sowohl eine Vollnarkose als auch das chirurgische Trauma können diese Balance zu Ungunsten des Immunsystems beeinflussen. Es ist bekannt, dass sowohl volatile Anästhetika als auch Opiate eine immunsupprimierende Wirkung besitzen.

Neuraxiale Verfahren können auf der einen Seite die inflammatorische Antwort eines chirurgischen Traumas reduzieren, auf der anderen Seite auch eine Einsparung von Hypnotika und Opiaten bewirken. Daher ist es naheliegend, sich in der Tumorchirurgie für eine Kombination von Vollnarkose mit Epiduralanästhesie zu entscheiden. Untersuchungen an Ratten und einzelne retrospektive Beobachtungsstudien haben diese Hypothese bestärkt.

\section{WAS WIR BEREITS ZUM THEMA WISSEN}

- Volatile Anästhetika und Opiate haben einen negativen immunmodulatorischen Effekt

- Ein neuraxiales Verfahren reduziert den chirurgischen Stress und bewirkt eine Einsparung an Anästhetika und Opiaten

- Im Tierversuch und in retrospektiven Untersuchungen zeigt sich eine Verbesserung des tumorfreien Intervalls

In der vorliegenden prospektiven randomisierten Beobachtungsstudie wurden 1.802 Patient*innen im Alter zwischen 60 und 90 Jahren wegen eines abdominellen oder thorakalen Tumoreingriffes entweder mit einer Vollnarkose (859) oder mit einer kombinierten Epidural- und Vollnarkose (853) anästhesiert. Die Vollnarkose wurde mittels Sevofluran mit oder ohne Lachgas und zusätzlichem Opiat (Remifentanil, Sufentanil, Fentanyl) durchgeführt. In der Gruppe mit der zusätzlichen PDA wurde Ropivacain verwendet.

In der primär veröffentlichten Studie erhielten die Patient*innen in der Kombinationsgruppe deutlich weniger
Nach einem mittleren Follow-up von 66 Monaten (5,5 Jahren) konnten noch 1.574 Patient*innen eingeschlossen werden. Nach diesem Zeitraum starben in der Kombinationsgruppe Vollnarkose plus Epiduralanästhesie 355 (42\%), in der Vollnarkosegruppe 326 (38\%; adjusted HR 1,07; $95 \%$ $\mathrm{Cl} 0,92-1,24 ; p=0,408)$. Es wurde auch kein signifikanter Unterschied im Event-Free Survival festgestellt. Somit konnte in dieser prospektiven Analyse kein Unterschied im Outcome zwischen einer Vollnarkose alleine oder kombiniert mit einer Epiduralanästhesie in der Tumorchirurgie festgestellt werden.

FAZIT für die Praxis: Diese Studie zeigt ein ernüchterndes Ergebnis hinsichtlich des Einflusses unserer Narkoseführung in der Langzeitmortalität und dem Event-Free Survival. Die Vollnarkose hat weder alleine noch in Kombination mit einer neuraxialen Blockade einen Einfluss auf TumorWiederkehr und Mortalität. Obwohl die Hypothese einer Verringerung der Beeinträchtigung des Immunsystems stimmig gewesen wäre und speziell tierexperimentelle Daten Anlass zur Hoffnung gegeben haben, ist es schwer zu erklären, warum eine deutliche Verringerung von Anästhetika und Analgetika nicht zu einer Reduktion der Tumor-Wiederkehr führte. Auffällig in dieser Studie sind lediglich die signifikanten Unterschiede im Perfusionsdruck und in der Herzfrequenz in der Kombinationsgruppe. Wir wissen von großen Kohortenstudien, dass eine Hypotension und eine Tachykardie unabhängige Risikofaktoren für eine Erhöhung der 1-Jahres-Mortalität sind. Ob dieser Effekt auch hier zum Tragen kommt, bleibt zu diskutieren. Danach wäre auch denkbar, dass eine konsequentere und striktere Einhaltung von hämodynamischen Parametern in der Kombinationsgruppe möglicherweise doch positive Effekte hervorruft.

Auch wenn das Argument einer Verringerung der Rückkehr des Tumors bei einer Vollnarkose + PDA nach dieser Datenlage schwer beizubehalten ist, kann doch bei bestimmten Patient*innen eine Kombination beider Verfahren sinnvoll sein. So wurde perioperativ festgestellt, dass eine Kombination eine Reduktion des postoperativen Delirs bewirkt. Speziell beim geriatrischen Patientengut kann eine Reduktion von Anästhetika und Opiaten zu einer verbesserten postoperativen Kognition führen. 